\title{
FuzzSemNIC: A Deep Fuzzy Neural Network Semantic-enhanced Approach of Neural Image Captioning
}

Tham Vo ( $\nabla$ thamvth@tdmu.edu.vn )

Thu Dau Mot University https://orcid.org/0000-0001-7291-4168

\section{Research Article}

Keywords: image captioning, neuro-fuzzy, RNN, attention

Posted Date: July 12th, 2021

DOl: https://doi.org/10.21203/rs.3.rs-610265/v1

License: (c) (i) This work is licensed under a Creative Commons Attribution 4.0 International License.

Read Full License 


\title{
FuzzSemNIC: a deep fuzzy neural network semantic- enhanced approach of neural image captioning
}

\author{
Tham $\mathrm{Vo}^{1, *}$ \\ ${ }^{I}$ Thu Dau Mot University, Binh Duong, Vietnam \\ 1thamvth@tdmu.edu.vn \\ ${ }^{(*)}$ Corresponding author
}

\begin{abstract}
Neural image captioning (NIC) is considered as a primitive problem artificial intelligence (AI) in which creates a connection between computer vision (CV) and natural language processing (NLP). However, recent attribute-based and textual semantic attention based models in NIC still encounter challenges related to irrelevant concentration of the designed attention mechanism on the relationship between extracted visual features and textual representations of corresponding image's caption. Moreover, recent NIC-based models also suffer from the uncertainties and noises of extracted visual latent features from images which sometime leads to the disruption of the given image captioning model to sufficiently attend on the correct visual concepts. To solve these challenges, in this paper, we proposed an end-to-end integrated deep fuzzy-neural network with the unified attention-based semantic-enhanced vision-language approach, called as FuzzSemNIC. To alleviate noises and ambiguities from the extracted visual features, we apply a fused deep fuzzy-based neural network architecture to effectively learn and generate the visual representations of images. Then, the learnt fuzzy-based visual embedding vectors are combined with selective attributes/concepts of images via a recurrent neural network (RNN) architecture to incorporate the fused latent visual features into captioning task. Finally, the fused visual representations are integrated with a unified vision-language encoder-decoder for handling caption generation task. Extensive experiments in benchmark NIC-based datasets demonstrate the effectiveness of our proposed FuzzSemNIC model.
\end{abstract}

Keywords: image captioning; neuro-fuzzy; RNN; attention;

\section{INTRODUCTION}

Recently, neural image captioning (NIC) [1] [2] [3] [4] has attracted a lot of attention from multiple researchers/organizations due to its potential applications in multiple disciplines. In general, image captioning is considered as a bridge which link the NLP and CV areas together in order to handle practical tasks of artificial intelligence domain in real-world applications, such as integrated visual linguistic retrieval, human-machine interaction (HMI), visual question answering (VQA) [5], etc. Specifically, recent NIC-based models are designed to automatically generate captions/descriptions in forms of natural language for user's input images which enable system to comprehensively perceive the world. The NIC is originally accelerated by dramatic developments of Internet, rich vision-based data generator (mobile/tablet devices) and online multimedia social networks (Instagram, Flickr, TikTok, etc.) in which a large number of visual data has been explosively created. The explosion of generated visual data on the Internet has been becoming an indispensable aspect today's big data, thus encourage the proposals of advanced CV-based models which can support to sufficiently understand and obtains valuable knowledge from these massive visual data resources. There are primitive baselines that have effectively contributed to the success of automatic image captioning tasks, include the visual object recognition, deep neural visual and linguistic representation learning. In early attempts of NIC, researchers have tried to learn and characterize the fused representations of images and their corresponding captions/descriptions in forms of textual data by applying complex sequential neural architectures which can be considered as the machine translation task. The basic idea of this approach is the model will receive the input representation of the image and perform a sequential 
representation learning for interpreting the visual features into the sequential predicted words in which the sequence-to-sequence (seq2seq) based architecture with the separated encoding and decoding mechanisms are used to handle this vision-to-text transformation process. Normally, traditional NIC models, such as: the well-known Google "show \& tell" (Google-ST) model [2], utilize a CNN-based encoder to learn the representation of input images then using a RNN-based architecture (GRU, LSTM, etc.) to generate the appropriate output caption/description. These RNN-based models are trained end-to-end using traditional back propagation method for optimizing model's parameters to maximize the likelihood of the predicting sequential caption words upon the given ground-truth caption content.

Along with tremendous raises of deep learning in both CV and NLP areas, complex neural architectures in both fields (CNN, neural seq2seq with attention [6] [7], transformer [8], etc.) which have been utilizes to automatically generate free-form fluent and linguistic natural captions/descriptions for the given images. Among recent advanced deep neural architectures, the proposal of attention-based mechanism in NLP has been widely applied to provide the system a capability of dynamically focusing on the latent relationships between specific image's regions and corresponding descriptive words in the caption. Hence, the visual attribute/feature-based attention mechanism is widely adopted in multiple recent image caption algorithms which demonstrate remarkable improvements in the quality of generated captions, more accurate and linguistic natural. In fact, feature/attribute visual attention is a critical mechanism in both image captioning and other visual human-machine interaction tasks. It plays an important role in designing NIC model to sufficiently interpret the captions/descriptions of images in forms of natural language can be biased towards the rich semantic and contextual representation. In particular, a NIC-based system with attribute/region attention-based mechanism is not designed to capture and describe all information in an image but just focus on important regions and detected objects inside.

\subsection{Recent achivements \& existing challenges in NIC}

Recently, visual feature/attribute attention-based mechanism is used to qualitatively provide the thorough concentration on the relationships between specific visual regions in images and sequential representation of descriptive text which enable the system to dynamically locate its gaze on the appropriate image's regions while generating the caption words. There are well-known models of this trend, such as: SemAtt [3], SCST [4] and AoANet [9]. Despite from significant improvements in the quality of generated captions in comparing with traditional simple RNN-based image captioning approach, visual attribute attention-based mechanism still encounter problems related to the misinterpretation between learnt visual attended attributes/regions appropriately with the sequential representations of image's textual description. In other words, attention-based NIC models mostly rely on the attribute (object, region, etc.) detection mechanism which is considered as independently working and unconnected with the natural linguistic captioning mechanism. Thus, the generated caption words sometime are irrelevant with the attended detected objects/regions inside an images. Thus, this problem might lead to the insufficient utilization of the timeconsuming rich contextual and semantic analysis steps. Moreover, partially considering the NIC is a neural machine translation mechanism, the incorporation of extracted attributes from an image in forms of visual attention-based contextual vectors is in some way considered as inappropriate with complex contextual representations of given training captions in form of human-written textual data. The irrelevance of the linguistic contextual information with the visual contexts of detected attributes/regions in image might sometimes lead to the misinterpretation of generated captions with the corresponding visual concepts in image. On the other side, researchers intend to deeply dive into the NLP domain [10] [11] [12] for finding other way for dealing with the semantic representation of generated captions in NIC task. Subsequently, there are rich semantic-enhanced attention strategies have been used to drive the system to more focus on the semantic importance of caption words on the provided visual representation of image without considering relationships between generative caption words with and attributes/featured regions of the given images. Thus, these approach still fail to comprehensively incorporate the linguistic contextual information of generated captions with the diversified learnt visual features. 
Moreover, recent CNN-based image encoding mechanisms which are used in recent NIC-based models also suffer problems related to the uncertainties and noises in the extracted visual latent features. The ambiguity in learnt visual features also leads to the downgrade of the generated caption quality in the after all. In fact, recent studies [13] [14] [15] have showed that the deep learning based architectures which are mainly applied for both CV and NLP areas normally suffer from data uncertainties and dramatic noises during the representation learning process. Along with the increase in both visual and textual data quantity, there are critical challenges regarding with data ambiguity has inevitably emerged that most of NIC-based systems may deeply involve in the high amount of noises and unpredictable uncertainties from extracted visual and textual features. These learnt data feature ambiguity challenges has imposed great burdensome on multiple AI tasks, including automatic image captioning.

\subsection{Our contributions}

To meet above listed challenges, we proposed a novel approach of integrated deep fuzzy-neural architecture with rich-semantic attention for image captioning task, called as FuzzSemNIC. In our proposed FuzzSemNIC model, we firstly apply a fused deep neuro-fuzzy network for image encoding task which is a combined Takagi-Sugeno-Kang (TSK)-based neuron-fuzzy architecture with the traditional deep CNNbased neural network. Our used fused fuzzy deep neural architecture is capable to reduce the noises and ambiguity in the learnt representations of images before utilizing them for the caption generation process. Then, combining with the extracted attributes in the given images, the fused visual information is sufficiently combined by using an RNN-based architecture with the attribute-based attention mechanism which is majorly inherited from the SemAtt model [3]. for handling caption generation task. To sum up, our contributions in this paper can be summarized as threefold, which are:

- First of all, the image representation learning process, we apply an integrated TSK-based fuzzy neural architecture with $\mathrm{CNN}$ to effectively learn the ambiguity-free latent feature representation of input images. Our applied fused fuzzy deep neural architecture (FDNN) [13] [14] in this paper is designed upon the principals of Takagi-Sugeno-Kang (TSK) fuzzy model which contains the inference and pooling operation layers. Then, the fuzzy-based learnt visual features are fused to the $\mathrm{CNN}$-based visual embedding vectors to form the final representations of a given image.

- Next, the learnt image representations which are achieved in previous step are used to combined with extracted attributes from the images and feed to a visual attention-based RNN-based architecture to handle the caption generation task. For extracted features from a given image, they are used to calculate the attention scores which support to control the caption word prediction in the given RNN architecture. Our used attribute-based attention mechanism in this paper is simply a combination of extracted visual attributes with the general representation of a given image in the sequential neural learning framework of RNN.

- Finally, to demonstrate the effectiveness of our proposed FuzzSemNIC model, we conducted extensive experiments in benchmark NIC-based datasets (MS-COCO and Flickr). The comparative experimental results demonstrate the outperformance of our proposed FuzzSemNIC model in comparing with recent state-of-the-art image captioning baselines.

The rest of our paper is organized into four sections. In the second section, we briefly review about recent studies and progresses in image captioning task, also discuss about pros/cons of recent models. Next, we formally present the methodology and implementation of our proposed FuzzSemNIC model in the third section. In fourth section, we demonstrate comparative experiments between FuzzSemNIC and recent image captioning baselines in benchmark datasets and discuss about experimental outputs. Finally, we conclude about our achievements in this paper and provide some potential improvements for future works.

\section{RELATED WORKS}

Google-ST ("shows and tell") [2] is considered an early approach of neural image captioning task which utilize the RNN-based architecture to learn and predict the caption words from the visual embedding 
information of input images by using CNN-based encoding mechanism. Most of earlier neural image captioning models [16] [17] [18] are considered as rule/template-based approach which are trained to generate the slotted caption words following the defined visual templates/patterns. However, templatebased image captioning models often suffer the limitations related to the generation of generic and irrelevant image caption due to the lack of thorough evaluation on the semantic representation of caption words as well as visual contextual information (related visual attributes/objects/concepts in the input images). Along the great achievements in advanced deep neural architecture for both CV and NLP areas. There are recent visual attention-based image captioning models have been proposed, such as: "semantic attention" - SemAtt [3], SCST [4] and AoANet [9] demonstrate remarkable improvements in the accuracy performance of image captioning task. In the SemAtt model, You, Q. et al. [3] proposed a novel approach of integrated visual attribute-based attention mechanism with RNN-based architecture to efficiently handle the image captioning task. The visual representation and extracted image's attributes (detected objects/regions) plays as the external controlling mechanism to force the generated caption words more focusing on the extracted visual attributes inside the input image. Similar to that in SCST model [4], Rennie, S. J. et al. also applied multiple visual attention-based mechanisms over the LSTM-based architecture to handle the caption generation task. In SCST model, Rennie, S. J. et al. also introduced the use of policy gradient upon selfcritical sequence training strategy of reinforcement learning framework to effectively optimize the given RNN-based architecture for image captioning task. Recently, keep going with the utilization of attention mechanism in image captioning, Huang, L. et al. proposed a novel dual attention mechanism called as: AoANet (2019) [9]. In general, the proposed AoANet is a neural encoder-decoder architecture which is quite similar with the seq2seq-based architecture for machine translation. In the AoANet model, Huang, L. et al. introduced an attention-on-attention module which is used in both encoder and decoder support for better modeling the relationships between extracted visual attributes and predicted caption words. However, these recent models still encounter challenges related to the feature uncertainties and noise reduction for the image representation learning process. Different from recent works, in our proposed FuzzSemNIC model, we apply a fused fuzzy deep neural network architecture to effectively learn the representation of inputs as well as reduces the uncertainties and noises from extracted visual features which are then used to facilitate the caption generation process via the visual attention-based RNN architecture.

\section{FuZzSEMNiC MODEL}

In this section, we formally present the methodology and detailed implementations of components in our proposed FuzzSemNIC model. For the initial image representation learning approach, we apply a fused fuzzy deep neural architecture (FDNN) to effectively learn the encoded visual information of input image. The use of different fuzzy membership function and rule-based layers in the given FDNN support to alleviate the feature noises and ambiguities from the learnt representations of images. Then, these achieved representations of the visual data are fed into an attention-based RNN architecture to fulfill the caption generation task.

\subsection{Fused fuzzy deep neural network (FDNN) for image representation learning}

\subsubsection{TSK-based deep fuzzy neural archiecture for fuzzy-based image representation learning}

First of all, to build up end-to-end fuzzy neural architecture for handling feature ambiguity-reduced representation learning task, we mainly apply the methodology of Takagi-Sugeno-Kang (TSK)-based fuzzy model which contains two major operations, include: fuzzy inferencing and pooling operation processes. For the fuzzy inference operation process, we use the strategy of single-in - multiple-outs based $(\mathrm{K})$ number of rules, which is formally defined as the condition with (n) number of outputs, as: if $x$ is $A_{k}$ then: $\left(\begin{array}{c}f_{1 k}(x) \\ \ldots \\ f_{n k}(x)\end{array}\right)$.

In our case, each single input $(\mathrm{x})$ is an image, then each $\mathrm{k}^{\text {th }}$ fuzzy rule is applied to capture the visual pattern of a specific $\mathrm{i}^{\text {th }}$ region/sub-region, denoted as: $\mathrm{r}_{\mathrm{i}}$, of the given image and each $\mathrm{i}^{\text {th }}$ mapping output, as: $\mathrm{f}_{\mathrm{ik}}(\mathrm{x})$ 
is a non-linear function over the specific region/sub-region of image. For the general rule-based calculation, the final output, as: $\mathrm{y}_{\mathrm{i}}$ of the mapping function $\mathrm{f}_{\mathrm{ik}}(\mathrm{x})$ is defined upon a specific fuzzy set, denoted as: $\mathcal{A}_{\mathrm{k}}$, as the following (as shown in equation 1):

$$
\begin{gathered}
\mathcal{P}_{\mathrm{k}}=\left[\mathrm{r}_{\mathrm{i}} \cdot \mathcal{A}_{\mathrm{k}}\right] \text { and } \overline{\mathcal{P}_{\mathrm{k}}}=\frac{\mathcal{P}_{\mathrm{k}}}{\sum_{\mathrm{j}=1}^{\mathrm{K}} \mathcal{P}_{\mathrm{j}}} \\
\mathrm{y}_{\mathrm{i}}=\sum_{\mathrm{k}=1}^{\mathrm{K}} \overline{\mathcal{P}_{\mathrm{k}}} \cdot \mathrm{f}_{\mathrm{ik}}(\mathrm{x})
\end{gathered}
$$

Similar to the traditional CNN-based approach, the region/sub-region of a given image is defined as the sliding window of the predefined filter (as illustrated in Figure 1). The fuzzy pooling strategy procedure is also implemented similarly to the inference process in which the fuzzy membership matrices are calculate upon a set of regions/sub-regions, then convolve the given image with the same size as fuzzy sets upon the defined filters (as illustrated in Figure 2).

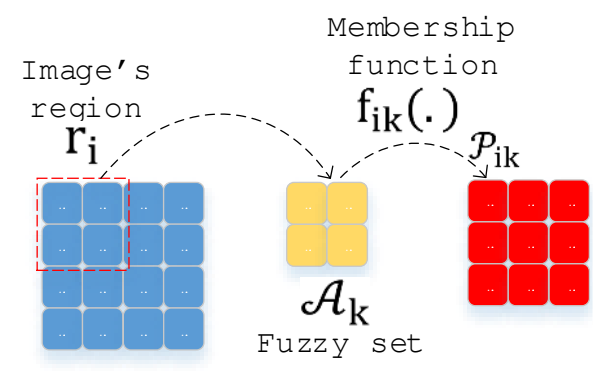

Figure 1. Illustration of the fuzzy membership calculation process

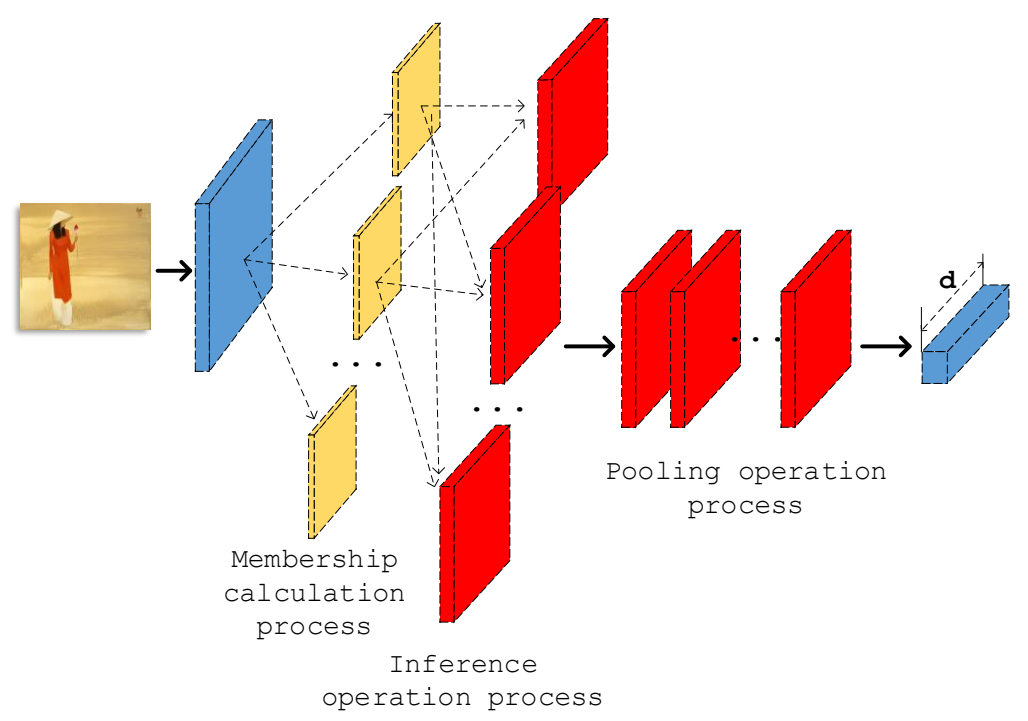

Figure 2. Illustration of overall TSK-based deep fuzzy neural network which is used in our paper

In after all, the completed TSK-based deep neuro-fuzzy architecture is composed by stacking all the fuzzy inference and operation processes. Then, at the end of this deep neuro-fuzzy architecture, the flatten strategy is applied to transform a given image to a fixed d-dimensional embedding vector, denote as: $\overrightarrow{\imath^{\mathrm{TSK}}} \in \mathbb{R}^{1 \times \mathrm{d}}$. Similar to the classical CNN-based architecture with the adding of inference and pooling operation in multiple fuzzy membership function and rule-based mapping layers, the applied TSK-based deep neural architecture is capable to reduce the noises and uncertainties from extracted features of a given image. 


\subsubsection{Fused fuzzy deep neural network (FDNN) for unifed fuzzy-deep image representation learning}

Then, to sufficiently merge previous obtained learnt d-dimensional TSK-based fuzzy representation of a given image with other the deep neural network based representation which is achieved by using pre-trained CNN-based image encoding models (e.g., Oxford-VGG [19], Microsoft-ResNet [20], etc.), we apply a hierarchical multi-layered fused fuzzy neural architecture to handle this fusion mechanism. Specifically, for each $\mathrm{i}^{\text {th }}$ input variable, denoted as: $\mathrm{x}_{\mathrm{i}}^{\text {fuzz }}$ of the given input TSK-based fuzzy embedding vector, as: $\overrightarrow{\imath^{\text {TSK }}}$ which is transformed a fuzzy membership layer in which each fuzzy neuron is defined as the mapping function at a specific $\mathrm{l}^{\text {th }}$ membership layer, as: $\mathrm{f}_{\mathrm{mem}}^{[1]}($.$) . This fuzzy membership mapping layer is used to$ transform a specific input variable, as: $x_{i}^{\text {fuzz,[1] }}$ into the fuzzy degree, denoted as: $y_{i}^{[1]}$ as the following (as shown in equation 2):

$$
\begin{gathered}
y_{i}^{\text {fuzz, }[0]}=f_{\text {mem }}^{[0]}\left(x_{i}^{\text {TSK }}\right)=e^{\frac{-\left(x_{i}^{\text {TSK }}-\mu_{i}\right)^{2}}{\sigma_{i}^{2}}}, \forall i \\
y_{i}^{f u z z,[1]}=f_{\text {mem }}^{[l]}\left(x_{i}^{\text {fuzz, [l] }}=y_{i}^{\text {fuzz, }[1-1]}\right)
\end{gathered}
$$

In each $1^{\text {th }}$ membership layer, the Gaussian function is used with the $\mu$ mean and $\sigma^{2}$ variance in which each fuzzy neuron is linked to the rule-based layer and performs the "AND" fuzzy logic operation. In the after all, the outputs of this fuzzy neural architecture is the fuzzy degrees of each input variable. On the deep neural architecture side, the visual embedding vectors of images, denoted as: $\overrightarrow{\imath^{\text {deep }}}$ which are achieved by using pre-trained CNN-based encoders [5] [6]. Then similar to the fuzzy-based neural architecture, for a specific $\mathrm{I}^{\text {th }}$ layer, each input variable of $\imath^{\mathrm{deep}}$, denoted as: $\mathrm{x}_{\mathrm{i}}^{\mathrm{deep},[1]}$ is separately fed into the full-connected dense-based layers with the sigmoid activation function in each layer. The overall process of this deep neural architecture part is defined as the following (as shown in equation 3):

$$
\begin{gathered}
x_{i}^{\text {deep,[l] }}=W^{\text {deep, }[1]} \cdot y^{[l-1]}+b_{i}^{\text {deep, }[1]} \\
y_{i}^{\text {deep, }[1]}=\sigma\left(x_{i}^{\text {deep, }[1]}\right)=\frac{1}{1+e^{-x_{i}^{\text {deep, }[1]}}}
\end{gathered}
$$

Defining as the dense linear layer, we have $\mathrm{W}^{\text {deep }}$ and $\mathrm{b}^{\text {deep }}$ are the trainable weighting and bias parameter matrices of each $\mathrm{I}^{\text {th }}$ full-connected layer, respectively. In general, our used FDNN architecture contains the separated fuzzy and deep neural architecture parts which are designed to achieve the better latent feature representation of an input image by reducing the noise and ambiguity from the learnt visual features. Finally, to effectively merge the fuzzy-based and deep-based representations of a given image into a unified embedding space, we apply a dense-connected fusion mechanism in which the output of this mechanism is the final fused latent feature representation of a given image, as: $\vec{\imath}$. This fused representation is the combinations of fuzzy degrees and deep visual neural representations altogether. The overall processes of this fusion mechanism can be formulated as the following (as shown in equation 4):

$$
\begin{gathered}
\mathrm{x}^{\text {fuse }}=\mathrm{W}_{\alpha}^{\text {fuse }} \cdot \mathrm{y}^{\text {deep }}+\mathrm{W}_{\beta}^{\text {fuse }} \cdot \mathrm{y}^{\text {fuzz }}+\mathrm{b}^{\text {fuse }} \\
\vec{\imath}=\sigma\left(\mathrm{x}^{\text {fuse }}\right)
\end{gathered}
$$

This fusion mechanism is also designed as a full-connected neural layer with the sigmoid function at the end, for: $\mathrm{W}_{\alpha}^{\text {fuse }}, \mathrm{W}_{\beta}^{\text {fuse }}$ and $\mathrm{b}^{\text {fuse }}$ are trainable weighting and bias parameter matrices which are jointly optimized with the overall FuzzSemNIC model during the model learning process. 


\subsection{Attention-based RNN architecture for image captioning}

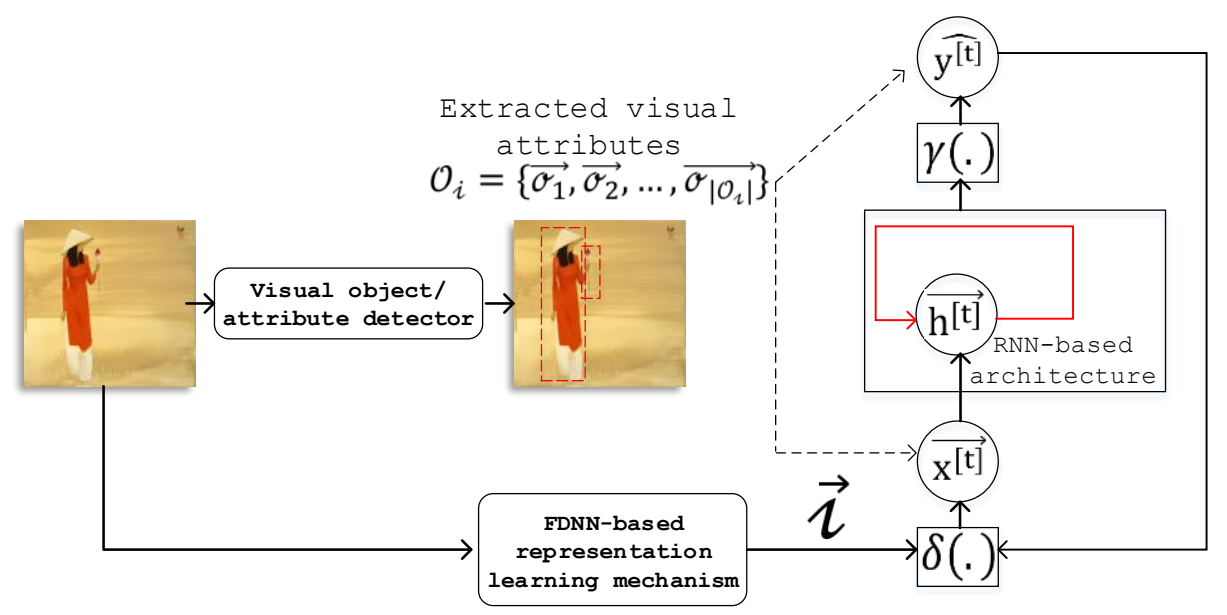

Figure 3. The illustration of our used visual attention-based RNN architecture in this paper

For each input image $(i)$, we apply a pre-trained visual attribute/object detector to extract a list of attributes, denoted as: $\mathcal{O}_{i}=\left\{\overrightarrow{\sigma_{1}}, \overrightarrow{\sigma_{2}}, \ldots, \overrightarrow{\sigma_{\left|O_{l}\right|}}\right\}$. In practical implementation, all the extracted visual attributes/concepts are encoded as one-hot vectors for the efficiency in computational processes. In our approach, the extracted visual attribute/concepts are forced to be associated with an entry (words/compound words) in the overall vocabulary set, denoted as: $\mathcal{V}$ for leveraging the performance of caption generation process. To do this, all the extracted visual attributes and fused visual representation of an image, as: $\mathcal{O}_{i}$ and $\vec{\imath}$, respectively are fed into a RNN-based architecture which is majorly inspired from the SemAtt model [3] (as illustrated in Figure 3). The fused fuzzy-based representation of image $(\vec{\imath})$ is fed into the RNN and played as the initial input to produce the starting state, denoted as: $\overrightarrow{x^{[0]}}=$ Linear $(\vec{\imath})=\mathrm{W}^{\text {init }} \cdot \vec{\imath}+\mathrm{b}^{\text {init }}$. At this stage, we apply a linear full-connected neural network to sufficiently map the visual latent representation of image into the given sequential learning mechanism of RNN, the trainable parameters: $\mathrm{W}^{\text {init }}$ and $\mathrm{b}^{\text {init }}$ are simultaneously optimized along with parameters of the given RNN architecture. In general, for a specific $\mathrm{t}^{\text {th }}$ time-step, the RNN generate the corresponding hidden state, as $\overrightarrow{h^{[t]}} \in \mathbb{R}^{1 \times d}$, a specific caption word is drawn from the given vocabulary set $(\mathcal{V})$ following the computed probabilistic distributions of $\widehat{y^{[t]}}$, in which: $\widehat{y^{[t]}} \in \mathbb{R}^{1 \times|\mathcal{V}|}$. Then, the predicted distribution on caption word, $\mathrm{y}^{[\mathrm{t}]}$, is recursively fed back again to the current RNN in the next $(t+1)^{\text {th }}$ time-step as a partial input, denoted as: $\mathrm{x}^{[\mathrm{t}+1]}$. All steps of this RNN-based training process can be formally illustrated as the following (as shown in equation 5):

$$
\begin{gathered}
\overrightarrow{\mathrm{h}^{[\mathrm{t}]}}=\operatorname{RNN}\left(\overrightarrow{\mathrm{h}^{[\mathrm{t}-1]}}, \overrightarrow{\mathrm{x}^{[\mathrm{t}]}}\right) \\
\overrightarrow{\mathrm{y}^{[\mathrm{t}]}} \sim \gamma\left(\overrightarrow{\mathrm{h}^{[\mathrm{t}]}}, \mathcal{O}_{i}\right) \\
\overrightarrow{\mathrm{x}^{[\mathrm{t}+1]}}=\delta\left(\widehat{\mathrm{y}^{[\mathrm{t}]}}, \mathcal{O}_{i}\right)
\end{gathered}
$$

The $\delta($.$) and \gamma($.$) are the input and output mapping functions, respectively of our given RNN-based$ architecture which enable to control the predicted words in each $\mathrm{t}^{\text {th }}$ time-step by the calculated attributebased scores. These two mapping functions are identified as the following (as shown in equation 6):

$$
\begin{gathered}
\alpha_{\mathrm{i}}^{[\mathrm{t}]} \propto \exp \left(\mathrm{y}^{\left[{ }^{[\mathrm{t}]}\right.} \cdot \mathcal{W}^{\mathrm{T}} \cdot \mathrm{U} \cdot \sigma_{\mathrm{i}}\right) \\
\delta\left(\widehat{\mathrm{y}^{[\mathrm{t}]}}, \mathcal{O}_{i}\right)=\mathrm{W}^{\delta}\left(\mathcal{W} \cdot \widehat{\mathrm{y}^{[\mathrm{tt}]}}+\operatorname{diag}\left(\mathrm{Z}^{\delta}\right) \cdot \sum_{\mathrm{i}} \alpha_{\mathrm{i}}^{[\mathrm{t]}} \mathcal{W} \cdot \overrightarrow{\sigma_{\mathrm{i}}}\right)
\end{gathered}
$$




$$
\begin{gathered}
\beta_{\mathrm{i}}^{[\mathrm{t}]} \propto \exp \left(\overrightarrow{\mathrm{h}^{[\mathrm{t}]}} \cdot \mathrm{V} \cdot \sigma\left(\mathcal{W} \cdot \overrightarrow{\sigma_{1}}\right)\right) \\
\gamma\left(\overrightarrow{\mathrm{h}^{[\mathrm{t}]}}, \mathcal{O}_{i}\right) \propto \exp \left(\mathcal{W}^{\mathrm{T}} \cdot \mathrm{W}^{\gamma} \cdot\left(\overrightarrow{\mathrm{h}^{[\mathrm{t}]}}+\operatorname{diag}\left(\mathrm{Z}^{\gamma}\right) \cdot \sum_{\mathrm{i}} \beta_{\mathrm{i}}^{[\mathrm{t}]} \cdot \sigma\left(\mathcal{W} \cdot \overrightarrow{\sigma_{\mathrm{i}}}\right)\right)\right)
\end{gathered}
$$

These two input/output mapping functions support to sufficiently model the relative importance of extracted visual attributes/concepts in each image to each time-step predicted caption words with $\mathrm{W}, \mathrm{Z}, \mathrm{U}, \mathrm{V}$ and $\mathcal{W}$ are the trainable weighting parameters of each mapping function and the word embedding matrices of all words in the given vocabulary set, $(\mathcal{V})$, as: $\mathcal{W} \in \mathbb{R}^{|\mathcal{V}| \times \mathrm{d}}$, respectively. In order to efficiently achieve the word embedding vectors of all words in the given vocabulary set, we can flexibly apply multiple recent pre-trained word representation learning models, such as: Word2Vec [21], GloVe [22], fastText [23] etc. To optimize model's parameters, denoted as: $\Theta^{\text {FuzzSemNic }}$, we apply the stochastic gradient descent (SGD) to minimize the negative log-likelihood loss which is identified as the following: $\min _{{ }_{\text {FuzzSemNic }}}-\sum_{\mathrm{t}} \log \left(\mathrm{y}^{[\mathrm{t}]}-\right.$ $\left.\widehat{y^{[t]}}\right)+\omega(\alpha)+\omega(\beta)$ with: $\omega($.$) is the regularization function which is identified as: \omega()=.\|\cdot\|_{1, p}+$ $\left\|\cdot{ }^{\mathrm{T}}\right\|_{1, q}-$ with: $p$ and $q$ are the predefined personalization constants.

\section{EXPERIMENTS \& DISCUSSIONS}

To demonstrate the effectiveness of our proposed FuzzSemNIC model in this paper, we conducted extensive experiments in benchmark datasets, including Microsoft-COCO and Flickr30K datasets. Comparative experimental results in terms of standard BLEU, Meteor, CIDEr and ROUGE-L metrics demonstrate the outperformances of our proposed FuzzSemNIC in comparing with recent image captioning baselines, such as: ST [2], SemAtt [3], SCST [4] and AoANet [9].

\subsection{Dataset description \& experimental setups}

\subsubsection{About MS-COCO and Flickr3OK datasets \& settings}

For all experiments in our paper, we used the MS-COCO and Flickr30K datasets to evaluate the performance of our proposed FuzzSemNIC model and other image captioning techniques. For the Flickr30K dataset, it contains 31,783 images with the corresponding 5 crowdsourced descriptions/captions. We applied the same split strategy of Karpathy, A. et al. [24] which used 29K images for training, $1 \mathrm{~K}$ for validation and $1 \mathrm{~K}$ for testing purposes. For the MS-COCO dataset, it is considered as a large-scale dataset with > 123,287 images (82,783 for training/ 40,504 for testing), and each image has at least 5 human-written captions/descriptions. We applied the same split strategy in previous works [3] to divide this dataset into three parts, as: training $(82,783)$, testing $(5 \mathrm{~K})$ and validation $(5 \mathrm{~K})$. Table 1 shows detailed information of the MS-COCO and Flickr30K datasets which are used in our experiments.

Table 1. General statistics of MS-COCO and Flickr3OK datasets which are used in our experiments

\begin{tabular}{|l|c|c|c|}
\hline \multicolumn{1}{|c|}{ Dataset } & Training & Validation & Testing \\
\hline MS-COCO & 82,783 & 5,000 & 5,000 \\
\hline Flickr30K & 29,783 & 1,000 & 1,000 \\
\hline
\end{tabular}

Textual pre-processing steps. For linguistic pre-processing steps on the caption contents of both Flickr30K and MS-COCO datasets, such as: word tokenization, stop-word filtering, stemming, etc. we main applied the Stanford-NLP library ${ }^{[1]}$ [25] to handle these steps. To efficiently learn the d-dimensional representations of all words in the vocabulary set of each dataset, we re-used the pre-trained GloVe [22]

${ }^{1}$ CoreNLP library for NLP (Java): https://stanfordnlp.github.io/CoreNLP/ 
word embedding model which is available for downloading at this repository ${ }^{[2]}$. We configured the word embedding vector dimensionality to 300 .

Configurations for FuzzSemNIC model. For the embedding vector dimensionality of the input image in our FDNN architecture (as described in section 3.1), we configured as 1024. To learn the initial deep neural representation of input images, we used the CNN-based pre-trained Microsoft ResNet-50 [20]. To extract bottom-up visual attributes from an input image, we applied the Fast-RCNN model [26]. For our image caption generation mechanism, we mainly used the LSTM for implementing the RNN-based architecture with the attribute-based attention mechanism as described in section 3.2. The number of used LSTM-based cells in the RNN-based architecture is set as 512. Table 2 demonstrates detailed configurations of our proposed FuzzSemNIC model for all experiments in this paper.

Table 2. Experimental configurations for FuzzSemNIC model

\begin{tabular}{|l|c|}
\hline \multicolumn{1}{|c|}{ Parameter of FuzzSemNIC model } & Value \\
\hline $\begin{array}{l}\text { Dimensionality of word embedding vector by } \\
\text { using GloVe model [22] }\left(\mathrm{d}^{\mathcal{W}}\right)\end{array}$ & 300 \\
\hline $\begin{array}{l}\text { Dimensionality of image embedding vector by } \\
\text { using FDNN approach (as described in section 3.1) } \\
\left(\mathrm{d}^{\text {FDNN }}\right)\end{array}$ & 1024 \\
\hline $\begin{array}{l}\text { Number of used LSTM cells (h) for the RNN- } \\
\text { based architecture }\end{array}$ & 512 \\
\hline Number of training epochs & 60 \\
\hline General learning rate $(\eta)$ & 0.001 \\
\hline Number of training batch size & 64 \\
\hline
\end{tabular}

\subsubsection{Comparative image captioning baselines}

To compare the performance of our proposed FuzzSemNIC model with recent image captioning baselines, we also implemented several state-of-the-art techniques in our experiments, which are:

- Google-ST (2015) [2]: is considered as an early attempt on the neural image captioning task which is firstly introduced by Vinyals, O. et al. In this work, Vinyals, O. et al. apply a simple integrated pre-trained CNN-base image encoder and RNN-based textual sequential generator to handle the image captioning task.

- SemAtt (2016) [3]: is a well-known semantic visual attribute attention-based image captioning model which is proposed by You, Q. et al. In the SemAtt model [3], You, Q., et al. introduced the use of visual attributes/concepts to facilitate the visual attribute attention-based RNN architecture for leveraging the quality of generated image caption. The ultimate purpose of using the attributebased attention mechanism is to control the generated caption words via RNN by using external visual attributes of the input image.

- SCST (2017) [4]: similar to SemAtt, the SCST is also a visual attribute attention-based image captioning approach which utilize different attention mechanisms (Att2In and Att2All) [4] within a LSTM-based architecture to significantly improve the accuracy performance of image captioning task. Different from previous approach, in the SCST model, Rennie, S. J. et al. [4] proposed a policy gradient with self-critical sequence training strategy as the reinforcement learning problem. Remarkable experimental outputs in MS-COCO dataset demonstrates the effectiveness of authors' ideas in the neural image captioning domain.

- AoANet (2019) [9]: in an recent advanced visual attribute attention-based mechanism for image captioning task. In the AoANet, Huang, L. el al. proposed a dual attention mechanism to fully capture the extracted visual information of input images within a neural encoder-decoder

\footnotetext{
${ }^{2}$ GloVe pre-trained word embedding model: https://nlp.stanford.edu/projects/glove/
} 
architecture to effectively model the relationships between extracted visual attributes and generated caption words in the given sequential encoder-decoder framework.

For detailed configurations in each comparative model which is mentioned above, we used the same described configurations in the original published paper in which it achieves the highest accuracy performance for the image captioning task. For other common settings, we configured them the same with our FuzzSemNIC model which are listed in Table 2.

\subsection{Experimental results \& discussions}

Automatic image captioning task. Table 3 and Table 4 show the experimental outputs in terms of BLUE (BLUE@1, BLUE@2,BLUE@3 and BLUE@4), METEOR ROUGE-L and CIDEr metrics of different image captioning models in the benchmark MS-COCO and Flickr30K datasets.

Table 3. Experimental outputs in terms of BLUE, METEOR, ROUGE-L and CIDEr standard metrics for automatic image captioning task in MS-COCO dataset

\begin{tabular}{|l|r|r|r|r|r|r|c|}
\hline & BLUE@ 1 & BLUE@2 & BLUE@ 3 & BLUE@ 4 & METEOR & ROUGE-L & CIDEr \\
\hline Google-ST & 0.66211 & 0.42671 & 0.20571 & 0.19821 & 0.25921 & 0.28912 & 0.68921 \\
\hline SemAtt & 0.71213 & 0.45786 & 0.37426 & 0.23429 & 0.26152 & 0.32567 & 0.98512 \\
\hline SCST & 0.78102 & 0.51921 & 0.47921 & 0.36921 & 0.27678 & 0.55921 & 1.00482 \\
\hline AoANet & 0.78215 & 0.58712 & 0.49821 & 0.38912 & 0.29391 & 0.58992 & 1.10181 \\
\hline FuzzSemNIC & $\mathbf{0 . 8 0 9 2 1}$ & $\mathbf{0 . 5 9 8 1 2}$ & $\mathbf{0 . 5 0 9 2 1}$ & $\mathbf{0 . 3 9 6 5 2}$ & $\mathbf{0 . 3 0 1 9 2}$ & $\mathbf{0 . 5 9 6 3 2}$ & $\mathbf{1 . 1 1 9 6 2}$ \\
\hline
\end{tabular}

Table 4. Experimental outputs in terms of BLUE, METEOR, ROUGE-L and CIDEr standard metrics for automatic image captioning task in Flickr30K

\begin{tabular}{|l|r|r|r|r|r|r|c|}
\hline & BLUE@1 & BLUE@2 & BLUE@ 3 & BLUE@ 4 & METEOR & ROUGE-L & CIDEr \\
\hline Google-ST & 0.63291 & 0.40032 & 0.27892 & 0.17821 & 0.16921 & 0.18921 & 0.58213 \\
\hline SemAtt & 0.65821 & 0.41921 & 0.28911 & 0.19821 & 0.19822 & 0.21921 & 0.87821 \\
\hline SCST & 0.71921 & 0.47212 & 0.32912 & 0.28213 & 0.26912 & 0.51721 & 0.98681 \\
\hline AoANet & 0.77821 & 0.52012 & 0.47821 & 0.31029 & 0.27891 & 0.54921 & 1.01921 \\
\hline FuzzSemNIC & $\mathbf{0 . 7 8 9 1 2}$ & $\mathbf{0 . 5 3 8 9 1}$ & $\mathbf{0 . 4 8 9 2 1}$ & $\mathbf{0 . 3 3 9 2 1}$ & $\mathbf{0 . 2 8 9 1 2}$ & $\mathbf{0 . 5 5 9 2 1}$ & $\mathbf{1 . 0 2 8 1 4}$ \\
\hline
\end{tabular}

As shown from experimental results, our proposed FuzzSemNIC explicitly achieves better performance than other baselines for image captioning task in both MS-COCO and Flickr30K datasets. In general, as shown from the experimental outputs in both datasets, most of visual attribute attention-based models (SemAtt, SCST, AoANet and our proposed FuzzSemNIC) significantly gain better performance than the traditional RNN-based model such as Google-NIC. The attention-based image captioning approach achieves better accuracy performance in term of CIDEr evaluation metric, approximately $52.75 \%$ and $68.01 \%$ in MS-COCO and Flickr30K datasets, respectively. In more details, in comparing with our main competitors in this experiment, which are: SemAtt, SCST and AoANet, our proposed FuzzSemNIC model also slightly improve the CIDEr-based accuracy performance about $15.26 \%$ (SemAtt), $7.83 \%$ (SCST) and $1.26 \%$ (AoANet) in both MS-COCO and Flickr30K datasets. This experimental outputs prove the facts that the use of fuzzy learning concept in the $\mathrm{CNN}$-based image representation learning process can effectively support to alleviate the feature noise and ambiguity from the learnt visual embedding vector which explicitly lead to the increase in the quality of generated image caption in after all.

\subsection{Ablation studies}

\subsubsection{Experimental studies on model's stability}


To study the stability of our proposed FuzzSemNIC model, we evaluated the accuracy performances of the FuzzSemNIC and our main competitor, AoANet model in handling image captioning task with different training set size (\%) of MS-COCO and Flickr30K datasets. Figure 6 shows the changes in accuracy performance in terms of CIDEr metric of our proposed FuzzSemNIC and AoANet models with different size of training set. The experimental outputs demonstrate the fact that both FuzzSemNIC and AoANet models are quite stable in the circumstance of size-varied training resources which indicates these two models can work well in case of having small size of training set. In more details, as shown from experiments in Flickr30K dataset (as shown in Figure 6-B), our proposed FuzzSemNIC model slightly reach better performance than AoANet within the dataset sizes in range 20\%-50\%.
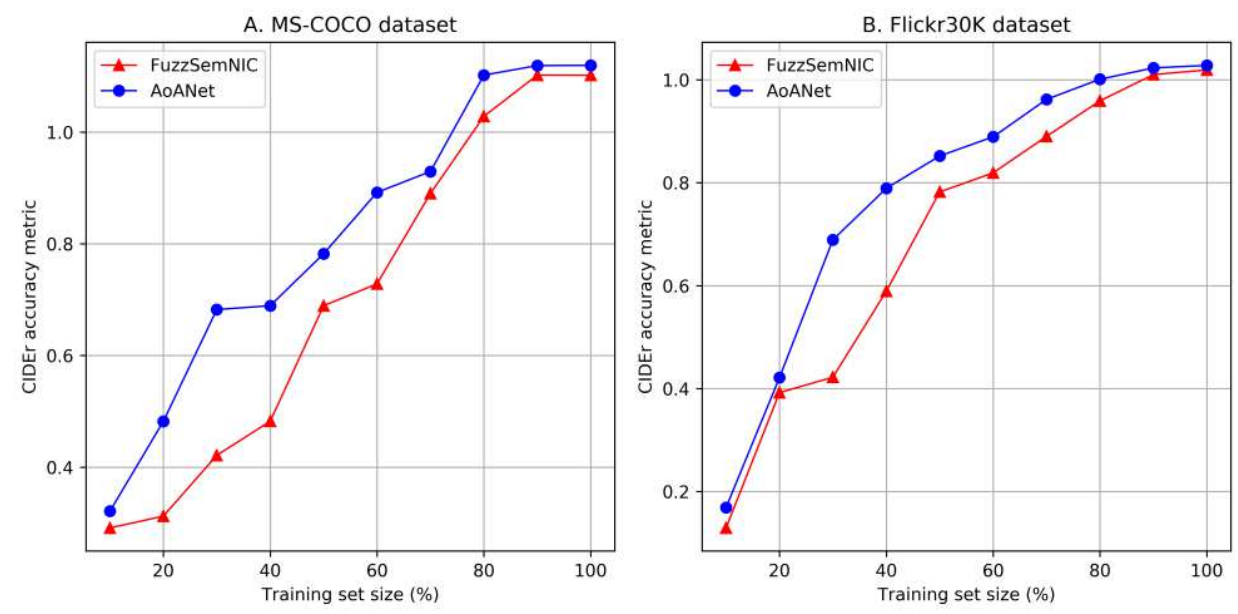

Figure 4. Experimental outputs in terms of CIDEr standard metric for image captioning task by using FuzzSemNIC and AoANet models with different training set sizes (\%) of MS-COCO and Flickr3OK datasets

\subsubsection{Model's parameter sensitivity analysis}
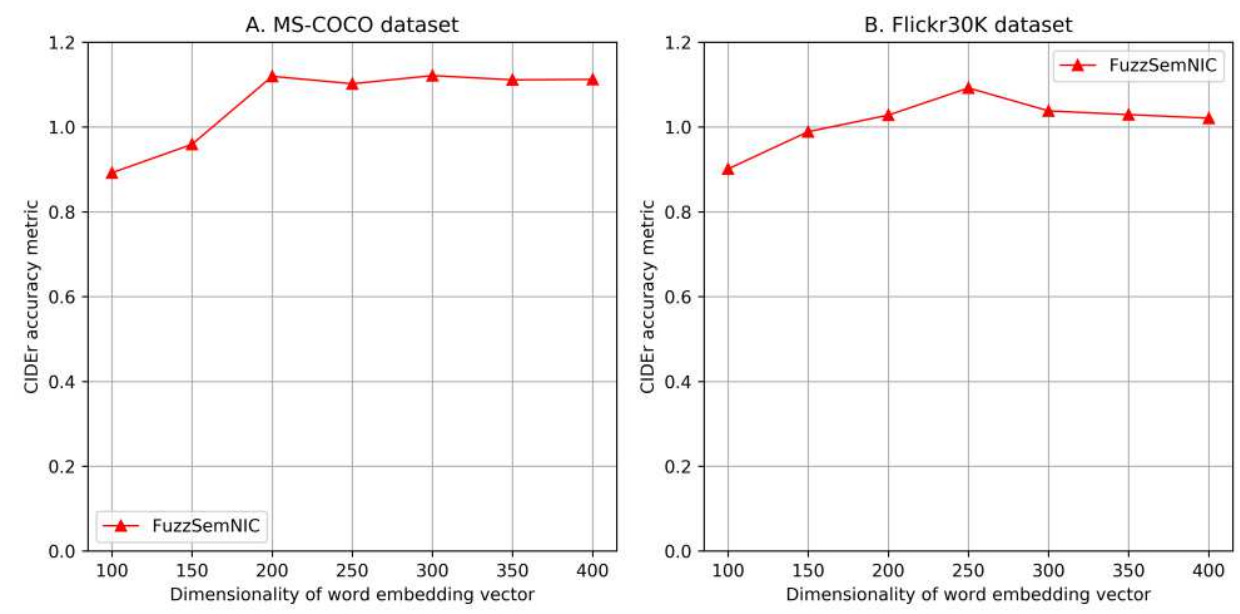

Figure 5. Experimental studies on the influence of word embedding dimensionality on the overall accuracy performance of our proposed FuzzSemNIC model in terms of CIDEr metric

In this section, we conducted extensive experiments to evaluate the effects of dimensionality of word embedding vector, as: $\left(\mathrm{d}^{\mathcal{W}}\right)$, which is achieved by using GloVe algorithm [8] and number of training epochs of our proposed FuzzSemNIC model. For the ablation study on the word embedding vector dimensionality, we varied the value of $\mathrm{d}^{\mathcal{W}}$ parameter in range of $[100,400]$ and reported the change of overall model accuracy performance in terms of CIDEr metric. As shown from experimental results in Figure 5, our proposed FuzzSemNIC model is considered as insensitive with the word embedding vector 
dimension parameter. With the value of $\mathrm{d}^{\mathcal{W}}$ parameter $>250$, our proposed FuzzSemNIC reach the highest accuracy performance in both MS-COCO and Flickr30K datasets. Thus, for practical implementation, the word embedding dimensionality can be easily adjusted upon the different sizes of the given vocabulary set.

Similar to the ablation study on $\mathrm{d}^{\mathcal{W}}$ parameter, in this experimental section, we evaluate the influence of configured number of training epochs of our proposed FuzzSemNIC over the MS-COCO and Flickr30K datasets. We implemented and trained our FuzzSemNIC model with different number of training epochs, in range [10-100] and reported the fluctuations of accuracy performance of image captioning task in terms of standard CIDEr metric.
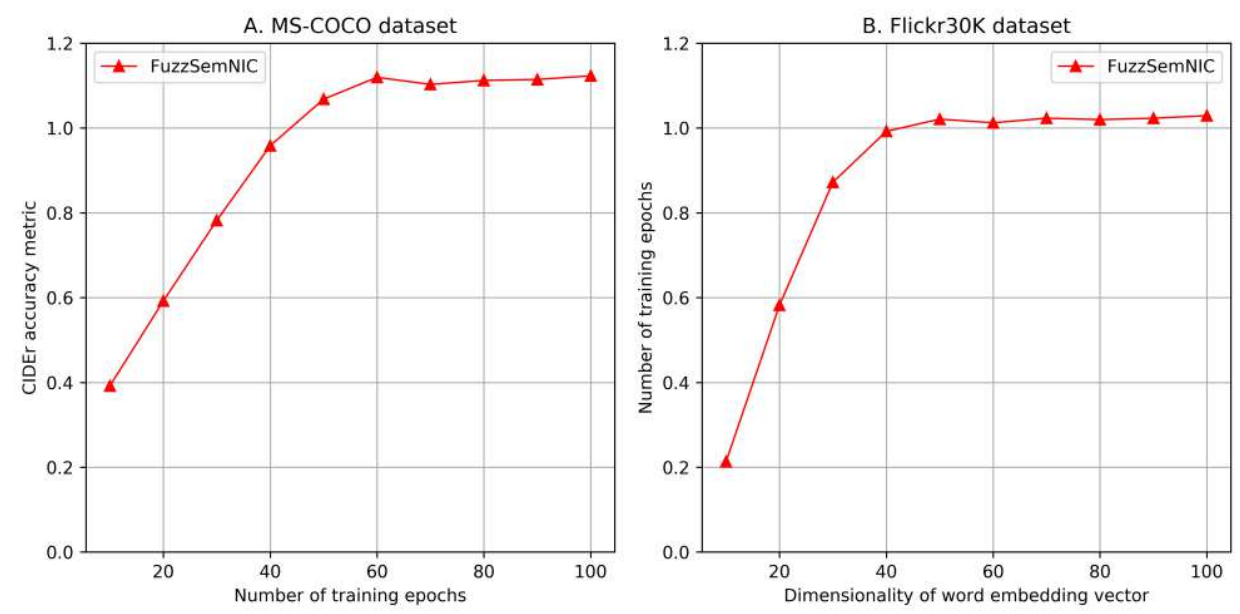

Figure 6. Experimental studies on the influence of number of training epochs on the overall accuracy performance of our proposed FuzzSemNIC model in terms of CIDEr metric

As shown from the experimental outputs in Figure 6, our proposed FuzzSemNIC model reach the convergent point with the number of training epochs $>60$ for both MS-COCO and Flickr30K datasets. This is considered as a reasonable training effort for both time-consuming and computational complexity optimizing in realistic implementation.

\section{CONCLUSIONS \& FUTURE WORKS}

In this paper, we proposed a novel approach of automatic image captioning task, called as FuzzSemNIC model. The proposed FuzzSemNIC is an integrated fused fuzzy deep neural network (FDNN) with the traditional CNN-based image representation learning for leveraging the performance of image captioning task. By applying the fuzzy learning concept in deep neural image encoding process, we can effectively reduce the noise and ambiguity from extracted visual features of input images. Then, the learnt fuzzy-based image representations are used to facilitate the RNN-based architecture with the visual attribute-based mechanism to efficiently handle the image caption generation process. Extensive experiments in benchmark MS-COCO and Flickr30K datasets demonstrate the effectiveness of our proposed FuzzSemNIC model in comparing with recent state-of-the-art image captioning baselines. For our future works, we plan to incorporate the masking language mechanism of BERT-based architecture [8] with the current visual attribute-based mechanism to improve the quality of generated image caption.

\section{DECLARATIONS}

This study was funded by Thu Dau Mot University, Binh Duong, Vietnam.

\section{ACKNOWLEDGEMENT}

This research is funded by Thu Dau Mot University, Binh Duong, Vietnam. 


\section{CONFLICT OF INTEREST}

This research is funded by Thu Dau Mot University, Binh Duong, Vietnam.

\section{REFERENCES}

[1] Kulkarni, G., Premraj, V., Ordonez, V., Dhar, S., Li, S., Choi, Y., ... \& Berg, T. L., "Babytalk: Understanding and generating simple image descriptions," IEEE Transactions on Pattern Analysis and Machine Intelligence, vol. 35, no. 12, pp. 2891-2903, 2013.

[2] Vinyals, O., Toshev, A., Bengio, S., \& Erhan, D., "Show and tell: A neural image caption generator," in Proceedings of the IEEE conference on computer vision and pattern recognition, 2015.

[3] You, Q., Jin, H., Wang, Z., Fang, C., \& Luo, J., "You, Q., Jin, H., Wang, Z., Fang, C., \& Luo, J.," in Proceedings of the IEEE conference on computer vision and pattern recognition, 2016.

[4] Rennie, S. J., Marcheret, E., Mroueh, Y., Ross, J., \& Goel, V., "Self-critical sequence training for image captioning," in Proceedings of the IEEE Conference on Computer Vision and Pattern Recognition, 2017.

[5] Antol, S., Agrawal, A., Lu, J., Mitchell, M., Batra, D., Zitnick, C. L., \& Parikh, D., "Vqa: Visual question answering," in Proceedings of the IEEE international conference on computer vision, 2015.

[6] Sutskever, I., Vinyals, O., \& Le, Q. V., "Sequence to Sequence Learning with Neural Networks," in Advances in Neural Information Processing Systems, 2014.

[7] Bahdanau, D., Cho, K., \& Bengio, Y., "Neural machine translation by jointly learning to align and translate," 3rd International Conference on Learning Representations, ICLR, 2015.

[8] Devlin, J., Chang, M. W., Lee, K., \& Toutanova, K., "Bert: Pre-training of deep bidirectional transformers for language understanding," in Proceedings of the 2019 Conference of the North American Chapter of the Association for Computational Linguistics: Human Language Technologies, 2019.

[9] Huang, L., Wang, W., Chen, J., \& Wei, X. Y., "Attention on attention for image captioning," in Proceedings of the IEEE/CVF International Conference on Computer Vision, 2019.

[10] Gan, Z., Gan, C., He, X., Pu, Y., Tran, K., Gao, J., ... \& Deng, L., "Semantic compositional networks for visual captioning," in Proceedings of the IEEE conference on computer vision and pattern recognition, 2017.

[11] Yu, N., Hu, X., Song, B., Yang, J., \& Zhang, J., "Topic-oriented image captioning based on orderembedding," IEEE Transactions on Image Processing, vol. 28, no. 6, pp. 2743-2754, 2018.

[12] Fang, F., Wang, H., \& Tang, P., "Image captioning with word level attention," in 25th IEEE International Conference on Image Processing (ICIP), 2018.

[13] Deng, Y., Ren, Z., Kong, Y., Bao, F., \& Dai, Q., "A hierarchical fused fuzzy deep neural network for data classification," IEEE Transactions on Fuzzy Systems, vol. 25, no. 4, pp. 1006-1012, 2016.

[14] Lin, F. J., Lin, C. H., \& Shen, P. H., "Self-constructing fuzzy neural network speed controller for permanent-magnet synchronous motor drive," IEEE transactions on fuzzy systems, vol. 9, no. 5, pp. 751-759, 2001. 
[15] Lin, C. T., Yeh, C. M., Liang, S. F., Chung, J. F., \& Kumar, N., "Support-vector-based fuzzy neural network for pattern classification," IEEE Transactions on Fuzzy Systems, vol. 14, no. 1, pp. 31-41, 2006.

[16] Devlin, J., Gupta, S., Girshick, R., Mitchell, M., \& Zitnick, C. L., "Exploring nearest neighbor approaches for image captioning," arXiv preprint arXiv:1505.04467, 2015.

[17] Wang, C., Yang, H., Bartz, C., \& Meinel, C., "Image captioning with deep bidirectional LSTMs," Proceedings of the 24th ACM international conference on Multimedia, pp. 988-997, 2016.

[18] Lebret, R., Pinheiro, P., \& Collobert, R., "Phrase-based image captioning," in International Conference on Machine Learning (PMLR), 2015.

[19] Simonyan, K., \& Zisserman, A., "Very deep convolutional networks for large-scale image recognition," in ICLR, 2015.

[20] He, K., Zhang, X., Ren, S., \& Sun, J., "Deep residual learning for image recognition," in Proceedings of the IEEE conference on computer vision and pattern recognition, 2016.

[21] Mikolov, T., Chen, K., Corrado, G., \& Dean, J., "Efficient estimation of word representations in vector space," in 1st International Conference on Learning Representations (ICRL), 2013.

[22] Pennington, J., Socher, R., \& Manning, C. D., "Glove: Global vectors for word representation," in Proceedings of the 2014 conference on empirical methods in natural language processing (EMNLP), 2014.

[23] Mikolov, T., Grave, É., Bojanowski, P., Puhrsch, C., \& Joulin, A., "Advances in Pre-Training Distributed Word Representations," in Proceedings of the Eleventh International Conference on Language Resources and Evaluation, 2018.

[24] Karpathy, A., \& Fei-Fei, L., "Deep visual-semantic alignments for generating image descriptions," in Proceedings of the IEEE conference on computer vision and pattern recognition, 2015.

[25] Manning, C. D., Surdeanu, M., Bauer, J., Finkel, J. R., Bethard, S., \& McClosky, D., "The Stanford CoreNLP natural language processing toolkit," in Proceedings of 52nd annual meeting of the association for computational linguistics: system demonstrations, 2014.

[26] Ren, S., He, K., Girshick, R., \& Sun, J., "Faster R-CNN: towards real-time object detection with region proposal networks," in Proceedings of the 28th International Conference on Neural Information Processing Systems, 2015. 